\title{
Performance of joint modelling of time-to-event data with time-dependent predictors: an assessment based on transition to psychosis data
}

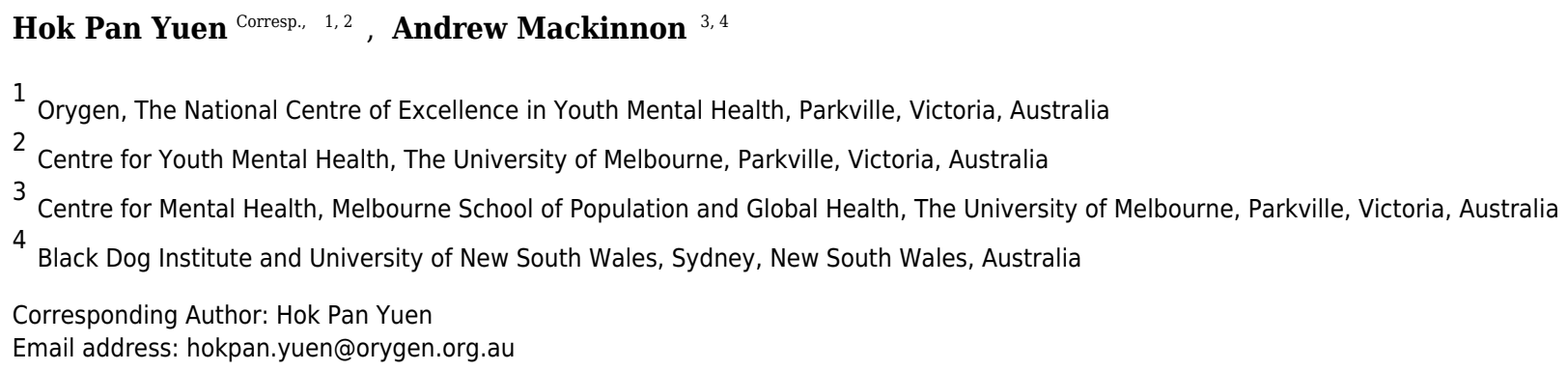

Joint modelling has emerged to be a potential tool to analyse data with a time-to-event outcome and longitudinal measurements collected over a series of time points. Joint modelling involves the simultaneous modelling of the two components, namely the timeto-event component and the longitudinal component. The main challenges of joint modelling are the mathematical and computational complexity. Recent advances in joint modelling have seen the emergence of several software packages which have implemented some of the computational requirements to run joint models. These packages have opened the door for more routine use of joint modelling. Through simulations and real data based on transition to psychosis research, we compared joint model analysis of time-to-event outcome with the conventional Cox regression analysis. We also compared a number of packages for fitting joint models. Our results suggest that joint modelling do have advantages over conventional analysis despite its potential complexity. Our results also suggest that the results of analyses may depend on how the methodology is implemented. 
1 Performance of joint modelling of time-to-event data with time-dependent predictors: an

2 assessment based on transition to psychosis data

3 Hok Pan Yuen ${ }^{1,2}$ and Andrew Mackinnon ${ }^{3,4}$

$4{ }^{1}$ Orygen, The National Centre of Excellence in Youth Mental Health, Locked Bag 10, Parkville,

5 Victoria, 3052, Australia.

$6{ }^{2}$ Centre for Youth Mental Health, The University of Melbourne, Parkville, Victoria, 3052,

7 Australia.

$8{ }^{3}$ Centre for Mental Health, Melbourne School of Population and Global Health, The University

9 of Melbourne, Parkville, Victoria, 3052, Australia.

$10{ }^{4}$ Black Dog Institute and University of New South Wales, Sydney, New South Wales, 2052,

11 Australia.

13 Corresponding author:

14 Hok Pan Yuen

15 Orygen, The National Centre of Excellence in Youth Mental Health,

16 Locked Bag 10, Parkville

17 Victoria 3052

18 Australia

19 Email address: hokpan.yuen@orygen.org.au

\section{Abstract}

21 Joint modelling has emerged to be a potential tool to analyse data with a time-to-event outcome

22 and longitudinal measurements collected over a series of time points. Joint modelling involves

23 the simultaneous modelling of the two components, namely the time-to-event component and the 
24 longitudinal component. The main challenges of joint modelling are the mathematical and 25 computational complexity. Recent advances in joint modelling have seen the emergence of 26 several software packages which have implemented some of the computational requirements to

27 run joint models. These packages have opened the door for more routine use of joint modelling, 28 in particular in the situation when there is one time-dependent predictor. Through simulations

29 and real data based on transition to psychosis research, we compared joint model analysis of 30 time-to-event outcome with the conventional Cox regression analysis. We also compared a

31 number of packages for fitting joint models. Our results suggest that joint modelling do have

32 advantages over conventional analysis despite its potential complexity. Our results also suggest

33 that the results of analyses may depend on how the methodology is implemented. 
35

36

37

\section{Introduction}

It is quite common in medical research to have a time-to-event variable as an outcome together with time-dependent predictors, which are basically longitudinal data collected on various characteristics. A typical situation is that the outcome is time to death and the time-dependent predictors are biomarkers which may be related to disease progression. Our group work in the youth mental health field in which a highly researched area is the so called ultra high-risk (UHR) patients [1-3]. These patients are assessed as being at high risk of becoming psychotic. In this scenario, the outcome is time to transition from a non-psychotic state to a psychotic state and the time-dependent predictors are various psychopathological and functioning measures.

The conventional approach used to analyse the kind of data mentioned above is the application of the Cox regression model. However, over the past two decades, methods that can provide a more flexible modelling framework for both the time-to-event and longitudinal aspects have emerged. The resulting models are named joint models. Extensive theory has been developed to provide a high level of flexibility to joint models such as allowing multiple timedependent predictors and allowing non-parametric, semi-parametric and parametric approaches [4-7]. However, the computational requirements for joint models is very demanding. In particular, multiple time-dependent predictors cannot be easily handled by available statistical software. In order to keep the scope manageable, this paper only focuses on the basic joint model with one time-dependent predictor. This paper has two aims. One aim is to assess the performance of joint modelling as compared to the conventional approach. Another aim is to compare the performance of a few joint modelling packages in a situation where the basic joint model is used with specifications that would typically be chosen by users of the respective software. These aims were achieved by simulating data which resembled transition to psychosis 
58 studies of UHR patients. The simulated data consisted of the times to transition, a baseline

59 predictor which was labelled as 'group' and a time-dependent predictor whose values

60 corresponded to monthly assessments over 12 months.

\section{The Cox regression model}

62 Time-to-event outcomes, such as time to death, time to relapse, time to discharge and so on, are common in medical research. Well established survival analysis methods are commonly used to analyse this type of outcome. Within the context of survival analysis, the Cox regression model [8] has been widely used to seek predictors or risk factors for time-to-event outcomes. The Cox model expresses the logarithm of the hazard rate as a linear function of the predictors. The hazard rate is a measure of the risk of the occurrence of the outcome event. So the Cox model relates the risk to the predictors. The estimated coefficient of each predictor is a measure of the effect of the predictor on the risk. In particular, the exponential value of the estimated coefficient can be regarded as a measure of the hazard ratio for a unit increase in the predictor concerned. There is a major difference between the Cox regression model and some other regression models, which often require an assumption about the underlying probability distribution of the outcome variable. For example, for the linear regression model, we usually need to assume that the outcome measure follows a normal distribution. The beauty about the Cox model is that we do not need to make any assumption about the underlying probability distribution of the outcome data and we can still estimate the effects of the predictors and test hypotheses about the predictors. The way that it achieves this is by using the method called partial likelihood [8-10]. This is one of the main reasons why the Cox model is so popular in the analysis of time-to-event data. 
80

81

82

83

84

85

86

87

The partial likelihood technique can be used when all the predictors are fixed as well as when some or all of the predictors are time-dependent. Fixed predictors are variables with fixed values for each individual. Examples are gender or age at baseline. Time-dependent predictors are variables with repeated measurements at a number of time points and their values may vary even for a particular individual. The execution of partial likelihood requires the values of the predictors at each recorded event time for all subjects concerned. This does not present any problem for fixed predictors because their values are constant. However, in most circumstances, measurements of time-dependent predictors are made at various assessment time points. Their values are usually not known at event times because the event times do not usually coincide with the assessment time points. A common way to overcome this problem is to employ the lastobservation-carried-forward approach and impute the unknown values with the corresponding last recorded values. This approach may not be reasonable if the time gap related to the imputation is large [11]. Also, parameter estimates and standard errors produced by this approach can be biased [12].

\section{Joint modelling}

Research has been carried out in the area of joint modelling since the mid-1990s [13] and is still an active research area. The joint modelling technique is designed to tackle the issues mentioned above in the Cox regression model with time-dependent predictors. The working of joint modelling is quite simple conceptually. Considering only one time-dependent predictor, it uses mixed-effects models [14] to estimate the trajectory or the trend of the time-dependent predictor and incorporates the estimated trajectory into the Cox regression framework [4]. Specifically, the hazard rate for subject $i$ at time $t$ is given by 


$$
h_{i}(t)=h_{0}(t) \exp \left(\gamma^{\mathrm{T}} \boldsymbol{\omega}_{i}+\alpha m_{i}(t)\right), i=1,2, \ldots, n, t>0 .
$$

104

Here $h_{0}(t)$ denotes the baseline hazard rate, $\omega_{i}$ is a vector of baseline predictors (e.g. treatment indicator, gender, age, etc.) and $\gamma$ is the corresponding vector of regression coefficients. The

107

108

109

110

111

112

113

114

115

116

117

118

119

120

121

122

123

124

125

time dependent predictor is represented by $m_{i}(t)$ with $\alpha$ being the corresponding coefficient

vector. A commonly used model for $m_{i}(t)$ is the linear mixed-effects model. Specifically, $m_{i}(t)$ is given by:

$$
\left.\begin{array}{c}
y_{i}(t)=m_{i}(t)+\varepsilon_{i}(t), \\
m_{i}(t)=\boldsymbol{X}_{i}^{T}(t) \boldsymbol{\beta}+\boldsymbol{Z}_{i}^{T}(t) \boldsymbol{b}_{i}, \\
\boldsymbol{b}_{i} \sim \mathrm{N}(0, \mathbf{D}), \varepsilon_{i}(t) \sim \mathrm{N}\left(0, \sigma^{2}\right) .
\end{array}\right\}
$$

Here $y_{i}(t)$ denotes the observed values of the time-dependent predictor for subject $i, \varepsilon_{i}(t)$ is an random error term, $\boldsymbol{\beta}$ is the vector of fixed effects and $\boldsymbol{b}_{\boldsymbol{i}}$ denotes the vector of random effects with covariance matrix $\mathbf{D}$. Both the random errors and the random effects are assumed to be normally distributed. The specifications in (2) imply that the time-dependent predictor is measured with error and that $m_{i}(t)$ is the corresponding 'true' value at time $t$. So in joint modelling the association between the event rate and the time-dependent predictor is modelled through the true values of the predictor.

The above specification can be regarded as the basic joint model [4] and is called the current value parameterization. Many variations to this basic specification of the joint model are possible [4]. For example, interaction terms between the baseline predictors and the time-dependent predictor can be introduced. There can be time-lagged time-dependent predictor, i.e. $m_{i}(t)$ becomes $m_{i}(t-c)$ where $c$ is the specified time lag. There can also be time-dependent slope, i.e. the derivative of $m_{i}(t)$. 
Parameters for joint models can be estimated using maximum likelihood [15-17] and well-

127 established algorithms such as the Expectation-Maximization (EM) algorithm [18] or the

128 Newton-Raphson algorithm [19]. Bayesian methods such as MCMC techniques can also be used

$129[20,21]$. In this paper, only maximum likelihood estimation is considered.

\section{Software for joint modelling}

131 Joint models can be fitted using the software R, Stata, SAS and WINBUGS [22]. For this paper,

132 the performance of the JM R package Version 1.4-0 [4, 23], the joineR R package Version 1.0-3

$133[24,25]$ and the stjm Stata module [26] were investigated.

134 The JM package is very versatile and allows many variations to the fitting of joint models.

135 Firstly, it allows the baseline hazard to be unspecified, to take the form of the hazard

136 corresponding to the Weibull distribution for the event times or to be approximated by (user-

137 controlled) piecewise-constant functions or splines. For ordinary Cox regression, the baseline

138 hazard is usually left unspecified. This is of course a well-known advantage of Cox regression.

139 This advantage avoids the restriction resulting from specifying a certain form for the baseline

140 hazard and at the same time still can offer valid statistical inference through the use of partial

141 likelihood. However, in the context of joint modelling, this advantage no longer holds because a

142 completely unspecified baseline hazard will generally lead to underestimation of the standard

143 errors of the model parameters [4, 16]. Although an unspecified baseline hazard function is one

144 of the options in the JM package, the recommendation is that one of the other options should be 145 used.

146 In addition to the current value parameterization, JM also allows for different specifications of 147 the time-dependent predictors such as time-lagged predictors and time-dependent slopes. It also 148 allows for an accelerated failure time [8] in place of the usual relative risk modelling framework 
149 for the survival model. The JM package relies on two other R packages: the survival package

150 [27] and the nlme package [28, 29]. The former is used to fit the Cox model and the latter is used

151 to fit the linear mixed-effects model. The package JM then extracts all the required information

152 (predictor vectors, design matrices, event indicator, etc.) from the two fitted models to fit the 153 joint model.

154 The package joineR assumes the Cox proportional hazard model for the time-to-event 155 outcome and it leaves the baseline hazard to be unspecified. The association between the time-to156 event and longitudinal components is based on the current value parameterization of the time157 dependent predictors. Three options for the specification of the random effects are allowed:

random intercept, random intercept and slope, and quadratic random effects. The standard errors of the model parameters are obtained by bootstrap, i.e. by re-estimating the model parameters from simulated realizations of the fitted model. So the above-mentioned issue of underestimated standard errors is not present in joineR. However, the time required to estimate the parameter standard errors can be relatively long because of bootstrapping.

The package stjm allows four options for the specification of the time-to-event outcome. The first three options make use of the proportional hazard model with the baseline hazard derived from the exponential, Weibull or Gompertz distributions. The fourth option utilizes the flexible parametric model, which is based on the cumulative hazard [26, 30]. Three options model the association between the time-to-event and longitudinal components: current value, timedependent slope and a time-independent structure linking the subject-specific deviation from the mean of the $k$ th random effect.

\section{Method}


171 As stated earlier, the Cox model can handle time-dependent predictors. Although it has some 172 potential limitations, its advantage is that it is relatively simple and well established. Joint

173 modelling can potentially overcome the limitations of Cox regression, but it is a much more

174 complicated methodology and is still at a relatively early stage of development. It is of interest to 175 compare the performance of the two. As joint modelling is more demanding computationally, it 176 is also of interest to investigate how the different joint modelling software packages compare. To 177 achieve these aims, a series of simulations were conducted. The details of these simulations are 178 given below.

179 Each simulation consisted of a time-dependent predictor, a time-to-event outcome and a group 180 variable of two levels. The time-dependent predictor was generated as follows:

181 (i) The timeframe was taken to be days $0,1,2, \ldots, 364$.

182 (ii) The value of the time-dependent predictor for subject $i$ at time $t(t=0,1,2, \ldots, 364)$ was 183 generated according to the following linear mixed-effects model:

$$
y_{i}(t)=a_{0}+a_{1} t+b_{0 i}+b_{1 i} t+\varepsilon_{i}(t)
$$

185 (iii) $a_{0}$ and $a_{1}$ were the fixed effects with given values.

186 (iv) $b_{0 i}$ and $b_{1 i}$ were the random effects generated from a bivariate normal distribution with mean $187 \quad 0$ and a given covariance matrix.

188 (v) $\varepsilon_{i}(t)$ was the random error generated from a normal distribution with mean 0 and a given 189 variance.

190 The time-to-event data was generated as follows:

191 (i) The hazard rate, $h_{i}(t)$, for subject $i$ at time $t(t=0,1,2, \ldots, 364)$, was computed as follows: 192

$$
h_{i}(t)=\exp \left(\lambda_{0}+\lambda_{1} m_{i}(t)+\tau u_{i}\right)
$$


where $m_{i}(t)$ denotes the unobserved true value of the time-dependent predictor (i.e. $a_{0}+a_{1} t+$ $\left.b_{0 i}+b_{1 i} t\right), \lambda_{0}$ and $\lambda_{1}$ are given values, $u_{i}$ is a group indicator ( 0 for group 1 and 1 for group 2$)$ and $\tau$ represents the group effect. In the above formulation, $\lambda_{l}$ is the effect of the timedependent predictor on survival. More specifically, $\exp \left(\lambda_{1}\right)$ is the hazard ratio for a unit

(ii) Based on (4), the hazard rate of subject $i$ can vary over time depending on the true value of the time-dependent predictor. But at a particular time $t, h_{i}(t)$ has a particular value and was taken to be the hazard from an exponential distribution. This allowed the generation of the time to event occurrence, $T_{i}$, for each subject.

(iii)The censoring time for each subject, $C_{i}$, was generated from a uniform distribution on the interval $[1,364]$.

(iv)If $T_{i} \leq C_{i}$, the survival status for subject $i$ was taken to be 1 and the time to event occurrence was taken to be $T_{i}$. Otherwise, the survival status was taken to be 0 and the censoring time was taken to be $C_{i}$.

To complete the generation of the simulated data, the data collection of the time-dependent predictor was taken to occur at regular time points, specifically at day 0 (i.e. baseline) and then at 30-day intervals thereafter. Also, the data of the time-dependent predictor was taken to be unavailable after the event time or censoring time, whichever was applicable. Therefore, for each subject, non-missing data for the time-dependent predictor were taken to be those at days 0,30 , 60 and so on up to the measurement occasion prior to the event time or censoring time. Any post-event or post-censoring data were not used.

214 The parameters associated with the simulations were given the following values:

(i) The fixed-effect intercept, $a_{0}$, was given the value 40 . 
216 (ii) The fixed-effect slope, $a_{1}$, was given two values, 0.02 and 0.1 . These two values were chosen

217 to contrast two different scenarios where one had a steeper trajectory than the other.

218 (iii)The covariance matrix of the random effects, $b_{0}$ and $b_{1}$, were given four different forms as

219 shown in Table 1. The four forms were chosen to represent different scenarios of bigger and

220 smaller variances and bigger and smaller correlations for the random effects.

221 (iv)The variance of the random error, $\varepsilon$, was given two values, 16 and 4 . The two values were

222 chosen to contrast bigger and smaller error variances.

223 (v) The hazard parameters, $\lambda_{0}$ and $\lambda_{1}$, were given the values -4.8 and -0.03 respectively.

224 (vi)The group effect, $\tau$, was given the value 0 . More simulations were done for which a non-zero

225 group effect was used. Details of these simulations are presented later in this paper.

226 Putting these parameter values together, there were 16 different sets of simulations ( $2 a_{l}$ values x

2274 random-effect covariance matrices $\times 2$ error variances $=16)$.

228 The above simulations pertain to a monotone pattern of data availability for the time-

229 dependent predictor, i.e. the time-dependent predictor is available for each subject from baseline

230 up until the subject is lost (due to event occurrence or censoring). While this pattern would still

231 be of interest for the purpose of simulation, it may not be very realistic. In practice, subjects may

232 miss some of the assessments at individual time-points in a haphazard manner. Also, the actual

233 assessment times in real studies may not always be at fixed intervals due to various practical

234 constraints. Because of these considerations, the above-mentioned 16 sets of simulations were

235 repeated for a non-monotone pattern of data for the time-dependent predictor with irregular

236 assessment times. This pattern was generated as follows:

237 (i) The data of the time-dependent predictor were again generated by the above-mentioned

$238 \quad$ linear mixed-effects model. 
239 (ii) Data collection for baseline was taken to always occur at day 0 . The nominal time-points for

240 subsequent data collection were again taken to be days 30,60 and so on. But the actual data

241 collection time-points for each subject were generated by adding a randomly generated

242 quantity to each of the nominal time-points. The quantity was an integer randomly chosen

243 from the interval $[-7,7]$. In other words, an assessment window of \pm 7 days was allowed for

244 each data collection time-point.

245 (iii)The number of post-baseline nominal data collection time-points was 12 (360/30). Of these

24612 , a randomly selected subset (of size ranging from 0 to 12) was chosen for each subject to

$247 \quad$ contain missing values.

248 (iv) The survival time was generated as mentioned earlier.

249 (v) The censoring time was taken to be the time of the last non-missing assessment unless the 250 generated event time was earlier.

251 In total 32 sets of simulation $(16 \times 2)$ were generated. For each set of simulation, 100 datasets

252 were generated. For each dataset, 150 subjects were generated for each of two groups, i.e. a total

253 of 300 subjects. The R package (version 3.1.1) [31] was used to produce the simulations.

254 The data generated from the above simulations resembled a transition to psychosis study in

255 which assessments are done at monthly intervals over a 12-month period [2]. The group variable

256 represents a fixed factor which can be a baseline characteristic such as gender or a treatment

257 factor in a clinical trial. The simulated values of the time-dependent predictor resembled those

258 of the Global Assessment of Functioning (GAF) Scale [32], the baseline values of which was

259 found to be a significant predictor of transition [33].

2606 Analyses of the simulated datasets 
261 As indicated above, joint modelling basically consists of a longitudinal sub-model and a survival

262 sub-model. To analyse the simulated datasets, joint modelling was applied with model (3) as the

263 longitudinal sub-model and model (4) as the survival sub-model. Whenever possible, a non-

264 parametric or semi-parametric approach was adopted for the specification of the baseline hazard

265 in order to avoid the need to choose a particular probability distribution. Also, for the purpose of

266 comparison, Cox regression using only the baseline value of the time-dependent predictor and

267 Cox regression using all non-missing values of the time-dependent predictor were also applied.

268 Specifically, for each dataset generated, the following analyses were carried out:

269 (i) The R survival package was used to fit Cox regression with the group variable and using only

270 the baseline values of the time-dependent predictor. This was done to provide information as

271 to whether there is any advantage to collect longitudinal measurements when the interest is

272 only on a time-to-event outcome. This can especially be useful when the aim is simply to

273 predict the occurrence of the time-to-event outcome. In other words, we are asking the

274 question: can we just take a simple approach and use the baseline values? This is, in fact, a

275 common approach in transition to psychosis studies probably due to its simplicity [33-35].

276 (ii) The R survival package was used to fit Cox regression with the group variable and the time-

277 dependent predictor (using the last observation carried forward approach as indicated earlier).

278 (iii)The joineR R package was used to fit joint model. As mentioned before, the baseline hazard

$279 \quad$ was unspecified in joineR.

280 (iv)The stjm Stata module was used to fit joint model. As stjm does not have the option of

281 leaving the baseline hazard unspecified in the basic joint model, the Weibull distribution was

282 chosen from the distributions available. 
283 (v) The JM R package was used to fit joint model with the baseline hazard specified to be a

284 piecewise-constant function, i.e. the baseline hazard was taken to have different values at different time intervals. As mentioned above, a completely unspecified baseline hazard in the context of joint modelling may lead to underestimation of the standard errors. So this semiparametric approach to the specification of the baseline hazard seeks to avoid the underestimation of the standard errors of the parameter estimates. At the same time, it allows some flexibility to the specification of the baseline hazard without the restriction of choosing a particular distribution.

(vi)The JM R package was used to fit joint model with the baseline hazard specified by regression splines this time. Specifically, the log baseline hazard is approximated using Bsplines. This is an alternative semi-parametric approach following the same rationale as using a piecewise-constant function.

The JM package offers two options for numerical integration: the standard Gauss-Hermite rule and the pseudo-adaptive Gauss-Hermite rule. It has been shown that the latter can be more effective than the former in the sense that typically fewer quadrature points are required to obtain an approximation error of the same magnitude and computational burden is reduced [4]. So the latter was used in the analyses using JM. For all other options in JM as well as in the other packages, the defaults were used in the analyses.

\section{Results}

The focus of this paper is on the estimation of the effect of the time-dependent predictor and also the group effect on survival. In other words, the focus is on the estimates of $\lambda_{1}$ and $\tau$, which were given the values of -0.03 and 0 respectively in the simulated datasets. In order to provide a visual presentation of these estimates, Figure 1 shows these estimates from one of the simulation 
306 sets in which the fixed effect slope $\left(a_{1}\right)$ is 0.02 , the error variance $(\varepsilon)$ is 16 and the random

307 effects covariance matrix takes the form: $\operatorname{Var}\left(b_{0}\right)=32, \operatorname{Var}\left(b_{1}\right)=0.002$ and $\operatorname{Cov}\left(b_{0}, b_{1}\right)=0.06$. For

308 this simulation set only, in addition to the analyses mentioned above, the JM package was also

309 used to fit joint model with an unspecified baseline hazard. The purpose of this extra analysis

310 was to gauge the potential underestimation of the parameter standard errors when the baseline

311 hazard is unspecified.

312 Figure 1a shows the boxplots of the estimates of $\lambda_{l}$ obtained from the 100 generated datasets.

313 It can be seen that there is substantial bias in the direction of underestimation in the Cox

314 regression using only the baseline value of the time-dependent predictor. A similar but less

315 substantial bias is also seen in the Cox regression using all of the longitudinal values of the time-

316 dependent predictor. A slight bias in the direction of over-estimation also appears in the joint-

317 modelling analysis using the JM package with a piecewise-constant baseline hazard. There

318 appears to be no bias in the results of all of the other joint-model analyses.

319 Figure $1 \mathrm{~b}$ shows the boxplots of the standard errors of the estimates of $\lambda_{1}$. The last boxplot in

320 this figure is for the JM joint model analysis with an unspecified baseline hazard. It demonstrates

321 the substantial underestimation of parameter standard errors for this approach. Figure 1c shows

322 the estimates of $\tau$. All approaches yielded similar estimates which appear to be unbiased. The

323 underestimation of parameter standard errors for the JM analysis with unspecified baseline

324 hazard was not observed for the standard errors of the estimates of $\tau$ (Figure 1d).

325 In order to provide a concise summary of the results of the 32 sets of simulations, we focus on the coverage of $95 \%$ confidence intervals and the degree of bias in estimation. The latter is

327 expressed as the percentage of estimates less than the true parameter value out of the 100

328 estimates for each set of simulation. If the estimation is unbiased, this percentage is expected to 
329 be around 50 (as illustrated in the boxplots of Figures 1a and 1c). Table 2 shows these results for 330 the estimation of $\lambda_{1}$ in each set of simulation. For the confidence intervals, it is expected that

331 good performance should correspond to a coverage of approximately $95 \%$, say $90 \%$ or more. For 332 unbiasedness, as mentioned above, the percentage of estimates less than the true parameter value 333 should be approximately 50 for good performance, say between 40 and 60. The shaded entries in 334 Table 2 are those scenarios which did not perform well. It can be seen that joineR and stjm 335 tended to show better results. Note also that, for a small number of the simulated datasets, the 336 estimates were not available when JM was used with a piecewise-constant baseline hazard due to 337 convergence problems.

338 The results in Table 2 are further summarized in Figure 2. In this figure, the points are plotted 339 as numbers with each number indicating the number of simulation sets (out of 32) with the 340 corresponding percentage. For example, the top number under Cox.b in Figure $2 \mathrm{a}$ is 3 with a 341 corresponding percentage of 97 . This indicates that 3 of the 32 simulated datasets had a coverage 342 of $97 \%$ when $95 \%$ confidence intervals were computed for $\lambda_{l}$ under Cox regression using only 343 the baseline values of the time-dependent predictor. Similarly, the top number under Cox.b in 344 Figure $2 \mathrm{~b}$ is 2 with a corresponding percentage of 63 . This indicates that $63 \%$ of the estimates for $345 \lambda_{1}$ were less than the true value for 2 out of the 32 datasets when Cox regression using only 346 baseline values were employed.

347 From Figure 2a, it can be seen that all of the datasets had coverage of more than $90 \%$ for $34895 \%$ confidence intervals under stjm. Similarly, all datasets except one had coverage of more 349 than $90 \%$ under joineR. For all other analysis methods, quite a number of datasets had coverage 350 less than $90 \%$, with a few much less than $90 \%$. 
percentage of estimates less than the true parameter value between 40 and 60. For Cox regression number of datasets had the percentage below 40 with some way below 40 . As the true value for $\lambda_{I}$ is negative, this suggests that these two analysis methods tended to underestimate or even reverse the value of $\lambda_{1}$. Conversely, for the two JM analyses, a substantial number of datasets had the percentage above 60 with some way above 60 . This suggests that these two analysis methods tended to overestimate the value of $\lambda_{1}$.

Figure 3 shows the corresponding results for the estimation of $\tau$. It can be seen from Figure 3a that all analysis methods had $90 \%$ or more for the confidence interval coverage. Figure $3 \mathrm{~b}$ shows that, except for a few occasions, the percentage of estimates less than the true parameter value were all between 40 and 60 for all the analysis methods. These results suggest that the performance of the different analysis methods were all good for the estimation of the group effect. repeated on four of the simulation sets for a non-zero group effect. These four sets were sets 3,7 , 19 and 23 in Table 2. These sets were chosen because they showed the worst results for the two 368 Cox analyses and the two JM analyses. The reason that a non-zero group effect was not applied to all the 32 simulation sets was that it was very time consuming to run the simulations. The nonzero group effect was taken to be -0.5 . Table 3 shows the results of these simulations, which are very similar to the corresponding results in Table 2. 
373 As a further assessment of the joint modelling methodology and the various software packages,

374 the same analyses described above were applied to a set of real data collected in a study of risk

375 factors of transition to psychosis in which a group of UHR patients were followed up for a

376 maximum period of 12 months [2]. Patients were recruited as they were admitted into the PACE

377 Clinic, which was an outpatient clinical service specifically developed to assess, manage and

378 follow up subjects at high risk of developing a psychotic disorder. Assessments were conducted

379 at study entry and subsequently at approximately monthly intervals until transition to frank

380 psychotic illness occurred, or until 12 months from study entry, whichever came first. For the

381 purpose of illustration, two potential risk factors were considered: family history of mental

382 illness and severity of depression. The former was ascertained as present/absent at entry,

383 indicating whether any first or second degree relatives had mental illness. The latter was

384 measured by the total score of the Hamilton Rating Scale for Depression (HAMD) [36] which

385 was administered on each occasion of measurement. Scores can range from 0 to 96 . Data were

386 available from 47 participants, 29 of whom had a family history of mental illness. The mean

387 HAMD score was 17.7 with an observed range of 2 to 39, representing negligible to severe

388 depression. The number of participants established as having transitioned to psychosis was 21 .

389 The time to transition ranged from 7 to 742 days from entry with a mean of 168 days (median

390118 days). For those who did not transition, the censoring time ranged from 339 to 707 days with

391 a mean of 450 days. (Transition time and censoring time could exceed 12 months from entry

392 because information from medical records were also used.)

393 The same analytic methods investigated above were applied to the data with family history of

394 mental illness as the group variable and depression score as the time-dependent predictor. The

395 results are shown in Table 4 . For the estimation of $\lambda_{l}$, it can be seen that the estimates can be 
396 divided into two groups - those based on the two Cox regressions and those based on the others.

397 The former are considerably smaller - they are about $60 \%$ of the others. This pattern appears to

398 agree with the results of the simulations, which showed that the two Cox regression methods

399 tended to underestimate $\lambda_{1}$. However, the tendency of the JM analysis to overestimate $\lambda_{1}$ as

400 observed in the simulations did not appear in this analysis. Neither did the tendency of the JM

401 analysis with unspecified baseline hazard to underestimate the standard error of $\lambda_{1}$. As the p-

402 values are usually the focus of clinical researchers, it is of interest to note that all the estimates of

$403 \lambda_{1}$ were significant at the 0.05 level, but the levels of significance varied widely.

404 For the estimation of $\tau$, while there were also substantial differences between estimates, the

405 standard errors were similar. It is again of interest to note that there was considerable variation

406 among the p-values, although all of them were non-significant at the 0.05 level.

\section{Discussion}

408 For time-to-event studies with longitudinal predictors, it is conceptually desirable to be able to

409 incorporate the longitudinal data in the prediction of the outcome event. Joint modelling is a

410 welcome addition to the set of analysis tools in such a situation. However, the implementation of

411 joint modelling is computationally challenging $[4,37,38]$. In fact, in the early days of joint

412 modelling, a two-stage approach was suggested $[39,40]$. The basic idea of this approach is to

413 firstly estimate the longitudinal trajectories using linear mixed-effects models. Then the

414 estimates obtained from this first stage are used in the survival models as observed values of the

415 predictors. Strictly speaking, this approach is not joint modelling because the two models are

416 fitted separately. Also, while such an approach is computationally less demanding, the resulting

417 estimates can be biased [41-44]. The main computational difficulty in joint modelling is that the

418 integrals involved in likelihood estimation are usually intractable in that they have no analytical 
419 solutions. So numerical approximations are usually required to evaluate these integrals. Much

420 research has been done in this aspect $[15,17,45-48]$. Bayesian methods for the estimation of

421 joint models have also been studied by various authors. But for the purpose of this paper, only

422 estimation based on maximum likelihood is considered.

423 Much progress has been made in devising techniques to resolve the computational issues

424 involved in joint modelling. As mentioned before, a number of software packages are already

425 available with some of these techniques implemented. However, as joint modelling is still an

426 emerging technique, it is certainly of interest to investigate its performance in practice. Wu et al

427 [37] conducted a simulation in which the longitudinal predictor followed a linear mixed-effects

428 model with both fixed and random intercepts and slopes. The covariance matrix of the random

429 effects was set to be diagonal and the baseline hazard was set to be constant. They compared the 430 joint-likelihood approach and the two-stage approach and found that the former produced less

431 biased estimates and more reliable standard errors than the latter. Ibrahim et al [49] conducted

432 simulation studies on joint modelling with the longitudinal data generated from a linear mixed-

433 effects model with random effects for an intercept and a slope and also a treatment effect

434 (equivalent to the group effect in this paper). The survival data were generated from the same 435 model as in (4). They found that, for the treatment effect on survival, both the Cox model with 436 the longitudinal data as a time-dependent covariate and the joint model gave nearly unbiased 437 estimates and gave similar performance in terms of confidence interval coverage. However, for a 438 non-zero effect of the longitudinal data on survival, they found that the former gave biased 439 estimates whereas the latter gave unbiased estimates. Also, the performance of the former was 440 less satisfactory than the latter in terms of confidence interval coverage. 
The results presented in this paper broadly agree with these simulation results in the sense that

442 joint modelling could perform better in terms of unbiasedness and confidence interval coverage,

443 especially in terms of the estimation of the effect of the time-dependent predictor on survival.

444 However it seems that the existence of these advantages may depend on how joint modelling is

445 implemented. The joineR package performed very well in our simulations. But its relative

446 weakness at this stage is that it is not very flexible as to how the joint model can be specified.

447 Also, as it utilizes bootstrap to obtain the parameter standard errors, it generally takes much

448 longer to run. For example, for each of the data sets considered in this paper (both simulated and

449 real), joineR took two to three minutes to run; whereas the other packages only took a few

450 seconds. The stjm package also performed quite well, but a potential limitation is that it does not

451 allow a completely unspecified baseline hazard.

452 Given that joint modelling allows better capture of the information of the changing values of

453 the longitudinal data over time, it would be expected that joint modelling would out-perform the

454 two Cox models considered in this paper especially in a situation when there is much error

455 variation in the longitudinal values. This can be seen in Table 2, which shows that the

456 performance of the two Cox models were especially poor when the error variance was large

$457(\operatorname{Var}(\varepsilon)=16)$. In such a situation, joint modelling should certainly be considered.

458 The analysis of the real data was consistent with the simulations in two respects. The first is

459 that different methodologies and different software may show non-trivial differences in results.

460 The second is that the two Cox-based models do appear to underestimate the parameter of the

461 time-dependent predictor. As this could have a substantive impact on interpretation of effects, it

462 is advisable to use these approaches with caution. 
463 In conclusion, the results in this paper support the conceptual and theoretical notions that joint 464 modelling has the potential to provide better statistical inference when time-to-event outcomes 465 are to be analysed with longitudinal data. The focus of this paper has been on the estimation of 466 the parameters associated with the time-to-event process. But joint modelling can also be utilized 467 to provide predictions for the survival probabilities, estimation for the longitudinal profile as well 468 as prediction for the time-dependent characteristics [4]. So it has the potential to be a powerful 469 and useful statistical tool. Since joint modelling is still a relatively new technique, further 470 development of corresponding statistical software is required. However, existing joint modelling 471 packages have already demonstrated their potential usefulness and should be utilized to apply 472 joint modelling in the analysis of relevant data, subject to the caveats raised in this paper. 
474

475

476

477

478

479

480

481

482

483

484

485

486

487

488

489

490

491

492

493

494

495

496

497

498

499

500

501

502

503

504

505

506

507

508

509

510

511

512

513

514

515

516

517

518

\section{References}

1. Yung AR, McGorry PD, McFarlane CA, Jackson HJ, Patton GC, Rakkar A. Monitoring and care of young people at incipient risk of psychosis. Schizophrenia bulletin. 1996;22(2):283303.

2. Yung AR, Phillips LJ, Yuen HP, Francey SM, McFarlane CA, Hallgren M, McGorry PD. Psychosis prediction: 12-month follow up of a high-risk ("prodromal") group. Schizophrenia research. 2003;60(1):21-32.

3. Yung AR, Phillips LJ, Yuen HP, McGorry PD. Risk factors for psychosis in an ultra highrisk group: psychopathology and clinical features. Schizophrenia research. 2004;67(23):131-42.

4. Rizopoulos D. Joint models for longitudinal and time-to-event data : with applications in R. Boca Raton: CRC Press; 2012.

5. Chi YY, Ibrahim JG. Joint models for multivariate longitudinal and multivariate survival data. Biometrics. 2006;62(2):432-45.

6. Tang N-S, Tang A-M, Pan D-D. Semiparametric Bayesian joint models of multivariate longitudinal and survival data. Comput Stat Data An. 2014;77:113-29.

7. Tang AM, Tang NS. Semiparametric Bayesian inference on skew-normal joint modeling of multivariate longitudinal and survival data. Statistics in medicine. 2015;34(5):824-43.

8. Kalbfleisch J, Prentice R. The Statisical Analysis of Failure Time Data. 2nd ed: Wiley; 2002.

9. Cox DR. Regression models and life tables (with discussion). Journal of the Royal Statistical Society Series B. 1972;34:187-220.

10. Cox DR. Partial likelihood. Biometrika. 1975;62:269-76.

11. Rizopoulos D, Takkenberg JJ. Tools \& techniques--statistics: Dealing with time-varying covariates in survival analysis--joint models versus Cox models. EuroIntervention : journal of EuroPCR in collaboration with the Working Group on Interventional Cardiology of the European Society of Cardiology. 2014;10(2):285-8.

12. Prentice RL. Covariate measurement errors and parameter estimation in a failure time regression model Biometrika. 1982;69(2):331-42.

13. Faucett CL, Thomas DC. Simultaneously modelling censored survival data and repeatedly measured covariates: a Gibbs sampling approach. Statistics in medicine. 1996;15(15):166385.

14. Verbeke G, Molenberghs G. Linear mixed models for longitudinal data. New York: Springer; 2009. xxii, 568 p. p.

15. Henderson R, Diggle P, Dobson A. Joint modelling of longitudinal measurements and event time data. Biostatistics. 2000;1(4):465-80.

16. Hsieh F, Tseng YK, Wang JL. Joint modeling of survival and longitudinal data: likelihood approach revisited. Biometrics. 2006;62(4):1037-43.

17. Wulfsohn MS, Tsiatis AA. A joint model for survival and longitudinal data measured with error. Biometrics. 1997;53(1):330-9.

18. Dempster AP, Laird NM, Rubin DB. Maximum likelihood from incomplete data via EM Algorithm. J Roy Stat Soc B Met. 1977;39(1):1-38.

19. Lange K. Optimization. New York: Springer; 2004. xiii, 252 p. p.

20. Brown ER, Ibrahim JG. A Bayesian semiparametric joint hierarchical model for longitudinal and survival data. Biometrics. 2003;59(2):221-8. 
519

520

521

522

523

524

525

526

527

528

529

530

531

532

533

534

535

536

537

538

539

540

541

542

543

544

545

546

547

548

549

550

551

552

553

554

555

556

557

558

559

560

561

562

563

564

21. Xu J, Zeger SL. Joint analysis of longitudinal data comprising repeated measures and times to events. J Roy Stat Soc C-App. 2001;50:375-87.

22. Gould AL, Boye ME, Crowther MJ, Ibrahim JG, Quartey G, Micallef S, Bois FY. Joint modeling of survival and longitudinal non-survival data: current methods and issues. Report of the DIA Bayesian joint modeling working group. Statistics in medicine. 2015;34(14):2181-95.

23. Rizopoulos D. JM: An R package for the joint modelling of longitudinal and time-to-event data. J Stat Softw. 2010;35(9):1-33.

24. Philipson P, Sousa I, Diggle PJ. joineR - joint modelling of repeated measurements and time-to-event data. 2012.http://cran.r-project.org/web/packages/joineR/vignettes/joineR.pdf.

25. Philipson P, Sousa I, Diggle P, Williamson P, Kolamunnage-Dona R, Henderson R. Package 'joineR'. 2015.http://cran.r-project.org/web/packages/joineR/joineR.pdf.

26. Crowther MJ, Abrams KR, Lambert PC. Joint modeling of longitudinal and survival data. Stata J. 2013;13(1):165-84.

27. Therneau T, Lumley T. Survival: Survival analysis including penalized likelihood. R package version 2.36-12. 2012.

28. Pinheiro J, Bates D, DebRoy S, Sarkar D. and R Development Core Team. nlme: Linear and nonlinear mixed-effects models. R package version 3.1-103. 2012.

29. Pinheiro JC, Bates DM. Mixed-effects models in S and S-PLUS. New York: Springer; 2000. xvi, 528 p. p.

30. Royston P, Parmar MKB. Flexible parametric proportional-hazards and proportional-odds models for censored survival data, with application to prognostic modelling and estimation of treatment effects. Statistics in medicine. 2002;21(15):2175-97.

31. R Core Team. R: A language and environment for statistical computing. $\mathrm{R}$ foundation for Statistical Computing. Vienna, Austria.2014.http://www.R-project.org/.

32. American Psychiatric Association. Diagnostic and Statistical Manual of Mental Disorders. 4th ed. Washington, DC: American Psychiatric Association; 1994.

33. Nelson B, Yuen HP, Wood SJ, Lin A, Spiliotacopoulos D, Bruxner A, Broussard C, Simmons M, Foley DL, Brewer WJ, Francey SM, Amminger GP, Thompson A, McGorry PD, Yung AR. Long-term follow-up of a group at ultra high risk ("prodromal") for psychosis: the PACE 400 study. JAMA psychiatry. 2013;70(8):793-802.

34. Cannon TD, Cadenhead K, Cornblatt B, Woods SW, Addington J, Walker E, Seidman LJ, Perkins D, Tsuang M, McGlashan T, Heinssen R. Prediction of psychosis in youth at high clinical risk: a multisite longitudinal study in North America. Archives of general psychiatry. 2008;65(1):28-37.

35. Ruhrmann S, Schultze-Lutter F, Salokangas RK, Heinimaa M, Linszen D, Dingemans P, Birchwood M, Patterson P, Juckel G, Heinz A, Morrison A, Lewis S, von Reventlow HG, Klosterkotter J. Prediction of psychosis in adolescents and young adults at high risk: results from the prospective European prediction of psychosis study. Archives of general psychiatry. 2010;67(3):241-51.

36. Hamilton M. A rating scale for depression. J Neurol Neurosurg Psychiatry. 1960;23:56-62.

37. Wu L, Liu W, Yi GY, Huang Y. Analysis of longitudinal and survival data: Joint modeling, inferencemethods, and issues. Journal of Probability and Statistics. 2012:17.

38. McCrink LM, Marshall AH, Cairns KJ. Advances in joint modelling: A review of recent developments with application to the sSurvival of end stage renal disease patients. International Statistical Review. 2013;81(2):249-69. 
565

566

567

568

569

570

571

572

573

574

575

576

577

578

579

580

581

582

583

584

585

586

587

588

589

590
39. Self S, Pawitan Y. Modeling a marker of disease progression and onset of disease. In: Jewell NP, Dietz K, Farewell VT, editors. AIDS Epidemiology: Methodological Issues. Boston: Birkhäuser; 1992.

40. Tsiatis AA, Degruttola V, Wulfsohn MS. Modeling the relationship of survival to longitudinal data measured with error - applications to survival and CD4 counts in patients with AIDS. J Am Stat Assoc. 1995;90(429):27-37.

41. Tsiatis AA, Davidian M. A semiparametric estimator for the proportional hazards model with longitudinal covariates measured with error. Biometrika. 2001;88(2):447-58.

42. Ye W, Lin XH, Taylor JMG. Semiparametric modeling of longitudinal measurements and time-to-event data-a two-stage regression calibration approach. Biometrics. 2008;64(4):1238-46.

43. Ratcliffe SJ, Guo WS, Ten Have TR. Joint modeling of longitudinal and survival data via a common frailty. Biometrics. 2004;60(4):892-9.

44. Sweeting MJ, Thompson SG. Joint modelling of longitudinal and time-to-event data with application to predicting abdominal aortic aneurysm growth and rupture. Biometrical J. 2011;53(5):750-63.

45. Song XA, Davidian M, Tsiatis AA. An estimator for the proportional hazards model with multiple longitudinal covariates measured with error. Biostatistics. 2002;3(4):511-28.

46. Rizopoulos D, Verbeke G, Molenberghs G. Shared parameter models under random effects misspecification. Biometrika. 2008;95(1):63-74.

47. Rizopoulos D, Verbeke G, Lesaffre E. Fully exponential Laplace approximations for the joint modelling of survival and longitudinal data. J Roy Stat Soc B. 2009;71:637-54.

48. Rizopoulos D. Fast fitting of joint models for longitudinal and event time data using a pseudo-adaptive Gaussian quadrature rule. Comput Stat Data An. 2012;56(3):491-501.

49. Ibrahim JG, Chu HT, Chen LM. Basic concepts and methods for joint models of longitudinal and survival data. J Clin Oncol. 2010;28(16):2796-801. 
592

593

1a. $\lambda_{1}$ estimates

594

595

596

597

598

599

600

601

602

603

604

605

606

607

608

609

610

611

612

613

614

615 1c. $\tau$ estimates

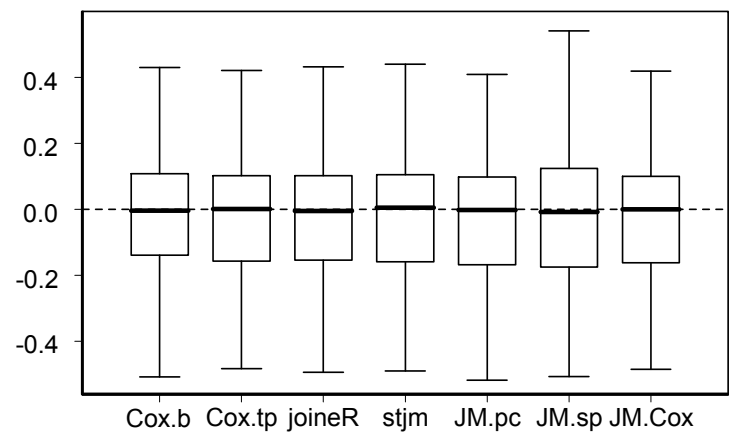

1b. s.e. $\left(\lambda_{1}\right.$ estimates $)$

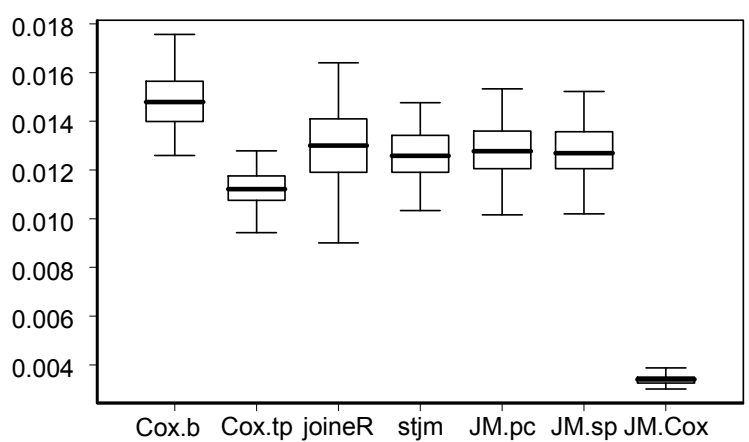

1d. s.e.( $\tau$ estimates $)$

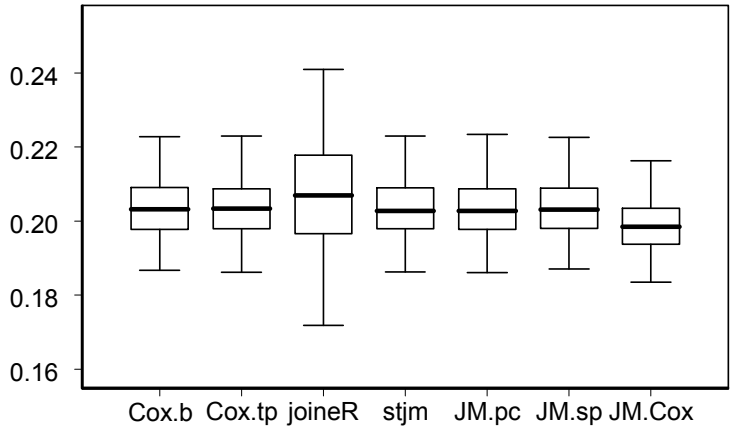

Figure 1. Boxplots of the results of the analysis of one of the simulation sets in which the fixed effect slope $\left(a_{1}\right)$ is 0.02 , the error variance $(\varepsilon)$ is 16 and the random effects covariance matrix takes the form: $\operatorname{Var}\left(b_{0}\right)=32, \operatorname{Var}\left(b_{1}\right)=0.002$ and $\operatorname{Cov}\left(b_{0}, b_{1}\right)=0.06$.

$\lambda_{1}$ : Parameter for the effect of the time-dependent predictor on survival (true value $=-0.03$ ).

$\tau$ : Parameter for the group effect on survival (true value $=0$ ).

Cox.b: Cox regression using only the baseline values of the time-dependent predictor.

Cox.tp: Cox regression using all of the longitudinal values of the time-dependent predictor.

JM.pc: JM package with a piecewise-constant baseline hazard.

JM.sp: JM package with the baseline hazard specified by regression splines.

JM.Cox: JM package with an unspecified baseline hazard. 
616

617

618

619

620

621

622

623

624

625

626

627

628

629

630

631

632

633

634

635

636

637

638

639

640

641 Figure 2. Graphical presentation of the results in Table 2.

642 2a: coverage of $95 \%$ confidence intervals.

643 2b: percentage of estimates less than the true parameter value out of the 100 estimates for each 644 set of simulation.

645 The points are plotted as numbers with each number indicating the number of simulation sets 646 (out of 32) with the corresponding percentage.

647 Refer to Figure 1 for other abbreviations. 
648

649

650

651

652

653

654

655

656

657

658

659

660

661

662

663

664

665

666

667

668

669

670

671

672

673

674

675

676

677

Estimation of $\tau$

3a. $95 \%$ C.I. coverage

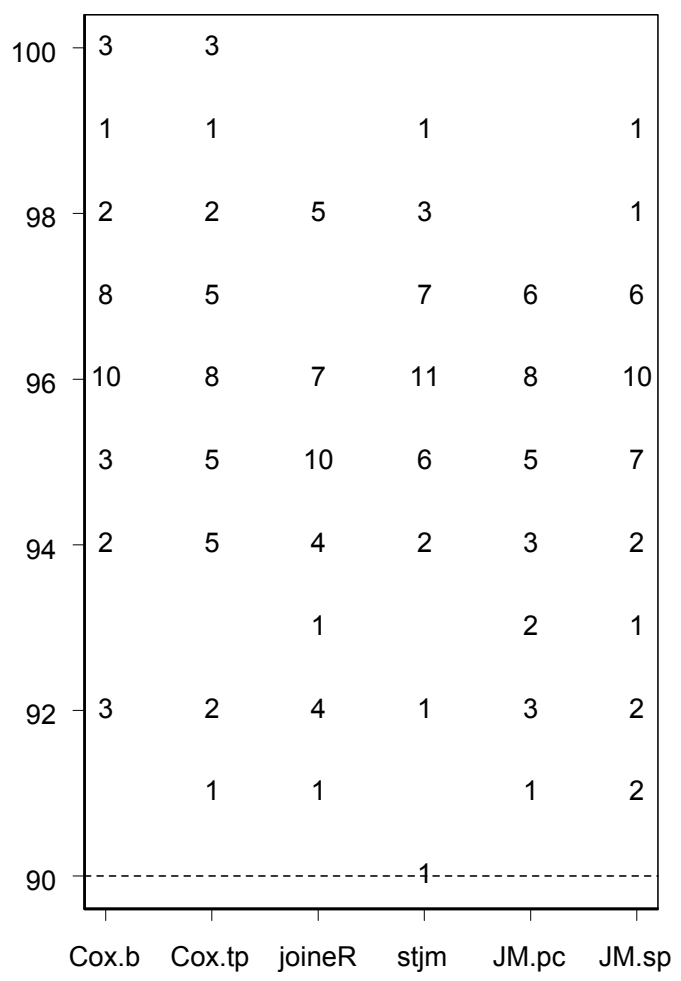

3b. $\%$ of estimates $<$ true value

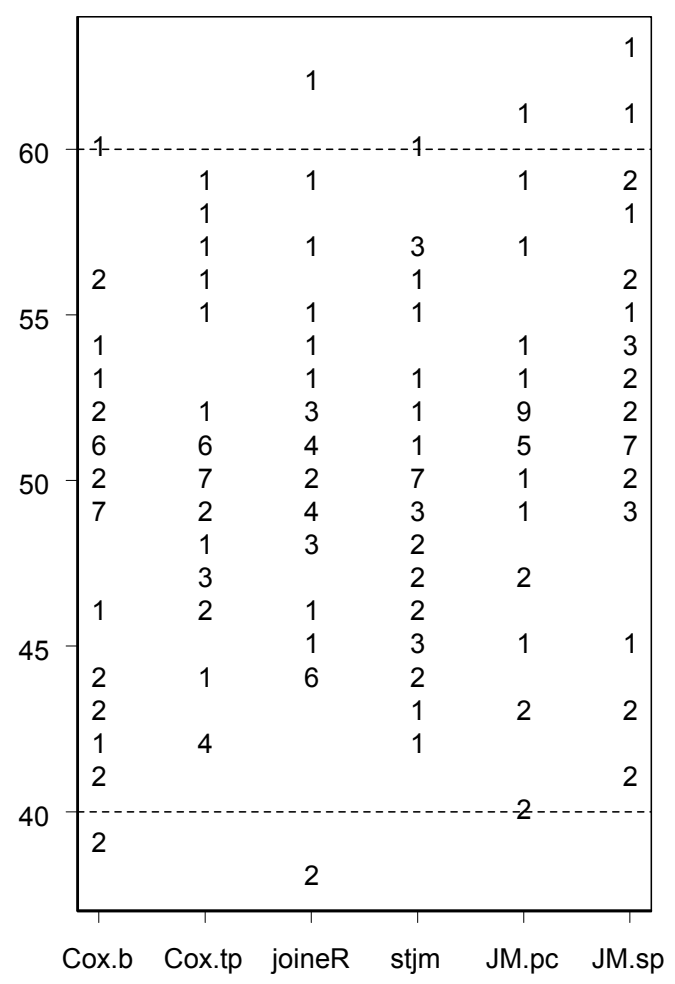

Figure 3. Graphical presentation of results of the estimation of $\tau$ from the 32 sets of simulations for which $\tau$ is zero.

Refer to Figures 1 and 2 for the meaning of the labels and the plots. 
679

680 Table 1. The four different forms used in simulations for the covariance matrix of the random 681 effects, $b_{0}$ and $b_{1}$, in Model (3).

\begin{tabular}{c|ccc|c} 
& $\operatorname{Var}\left(b_{0}\right)$ & $\operatorname{Var}\left(b_{1}\right)$ & $\operatorname{Cov}\left(b_{0}, b_{1}\right)$ & Correlation \\
\hline $\mathrm{a}$ & 32 & 0.002 & 0.06 & 0.237 \\
$\mathrm{~b}$ & 32 & 0.002 & 0.02 & 0.079 \\
$\mathrm{c}$ & 8 & 0.0005 & 0.02 & 0.316 \\
$\mathrm{~d}$ & 8 & 0.0005 & 0.002 & 0.032
\end{tabular}


Table 2. Results of the estimation of $\lambda_{1}$ from the 32 sets of simulations for which group effect is zero.

\begin{tabular}{|c|c|c|c|c|c|c|c|c|c|c|c|c|c|c|c|c|}
\hline \multirow[b]{2}{*}{$\begin{array}{l}\text { m.v. } \\
\text { pattern }\end{array}$} & \multirow[b]{2}{*}{$\operatorname{Var}(\varepsilon)$} & \multirow[b]{2}{*}{$a_{1}$} & \multirow[b]{2}{*}{$D$} & \multirow[b]{2}{*}{ Set } & \multicolumn{6}{|c|}{ Coverage of $95 \%$ confidence intervals } & \multicolumn{6}{|c|}{ Percentage of estimates $<$ true value } \\
\hline & & & & & Cox.b & Cox.tp & joineR & stjm & JM.pc & JM.sp & Cox.b & Cox.tp & joineR & stjm & JM.pc & JM.sp \\
\hline \multirow[t]{16}{*}{ Monotone } & \multirow[t]{8}{*}{16} & \multirow[t]{4}{*}{0.02} & $\mathrm{a}$ & 1 & 94 & 94 & 91 & 94 & 97 & 94 & 24 & 40 & 50 & 52 & 59 & 53 \\
\hline & & & $\mathrm{b}$ & 2 & 83 & 93 & 92 & 94 & 95 & 95 & 27 & 40 & 52 & 53 & 57 & 52 \\
\hline & & & $\mathrm{c}$ & 3 & 82 & 88 & 92 & 93 & 69 & 84 & 20 & 24 & 53 & 58 & 90 & 79 \\
\hline & & & $\mathrm{d}$ & 4 & 92 & 90 & 94 & 95 & 91 & 94 & 44 & 24 & 53 & 57 & 40 & 59 \\
\hline & & \multirow[t]{4}{*}{0.1} & $\mathrm{a}$ & 5 & 88 & 94 & 91 & 96 & 87 & 91 & 33 & 37 & 52 & 56 & 83 & 76 \\
\hline & & & $\mathrm{b}$ & 6 & 90 & 94 & 93 & 97 & 88 & 92 & 25 & 32 & 50 & 55 & 85 & 80 \\
\hline & & & $\mathrm{c}$ & 7 & 84 & 85 & 94 & 95 & NA & 96 & 19 & 24 & 47 & 58 & NA & 79 \\
\hline & & & $\mathrm{d}$ & 8 & 88 & 94 & 91 & 95 & 87 & 91 & 33 & 37 & 52 & 62 & 83 & 76 \\
\hline & \multirow[t]{8}{*}{4} & \multirow[t]{4}{*}{0.02} & $\mathrm{a}$ & 9 & 96 & 95 & 91 & 93 & 96 & 94 & 46 & 52 & 48 & 48 & 56 & 53 \\
\hline & & & $\mathrm{b}$ & 10 & 92 & 95 & 93 & 93 & 96 & 95 & 43 & 54 & 53 & 53 & 61 & 53 \\
\hline & & & $\mathrm{c}$ & 11 & 92 & 94 & 96 & 97 & 74 & 83 & 39 & 47 & 49 & 50 & 92 & 82 \\
\hline & & & $\mathrm{d}$ & 12 & 96 & 95 & 91 & 92 & 96 & 94 & 46 & 52 & 48 & 54 & 56 & 53 \\
\hline & & \multirow[t]{4}{*}{0.1} & $\mathrm{a}$ & 13 & 92 & 95 & 91 & 95 & 89 & 95 & 48 & 46 & 48 & 54 & 74 & 72 \\
\hline & & & $\mathrm{b}$ & 14 & 92 & 96 & 92 & 95 & 88 & 93 & 48 & 49 & 47 & 54 & 80 & 79 \\
\hline & & & $\mathrm{c}$ & 15 & 94 & 92 & 94 & 93 & NA & 97 & 35 & 42 & 47 & 60 & NA & 83 \\
\hline & & & $\mathrm{d}$ & 16 & 92 & 95 & 91 & 94 & 89 & 95 & 48 & 46 & 48 & 58 & 74 & 72 \\
\hline \multirow{9}{*}{$\begin{array}{l}\text { Non- } \\
\text { monotone }\end{array}$} & \multirow[t]{8}{*}{16} & \multirow[t]{4}{*}{0.02} & $\mathrm{a}$ & 17 & 97 & 96 & 93 & 93 & 96 & 90 & 33 & 31 & 60 & 57 & 44 & 58 \\
\hline & & & $\mathrm{b}$ & 18 & 87 & 94 & 88 & 94 & 94 & 93 & 23 & 40 & 49 & 50 & 51 & 53 \\
\hline & & & $\mathrm{c}$ & 19 & 85 & 84 & 95 & 95 & 91 & 73 & 21 & 25 & 53 & 53 & 79 & 90 \\
\hline & & & $\mathrm{d}$ & 20 & 97 & 96 & 93 & 91 & 96 & 90 & 33 & 31 & 60 & 63 & 44 & 58 \\
\hline & & \multirow[t]{4}{*}{0.1} & $\mathrm{a}$ & 21 & 95 & 92 & 94 & 95 & 95 & 91 & 33 & 24 & 45 & 55 & 63 & 81 \\
\hline & & & $\mathrm{b}$ & 22 & 90 & 88 & 91 & 92 & 95 & 91 & 29 & 21 & 50 & 54 & 62 & 80 \\
\hline & & & $\mathrm{c}$ & 23 & 84 & 77 & 94 & 94 & NA & 96 & 20 & 12 & 52 & 63 & NA & 89 \\
\hline & & & $\mathrm{d}$ & 24 & 95 & 92 & 94 & 97 & 95 & 91 & 33 & 24 & 45 & 59 & 63 & 81 \\
\hline & 4 & 0.02 & $\mathrm{a}$ & 25 & 94 & 95 & 91 & 95 & 96 & 94 & 58 & 61 & 56 & 58 & 57 & 57 \\
\hline
\end{tabular}


m.v. pattern: Missing value pattern.

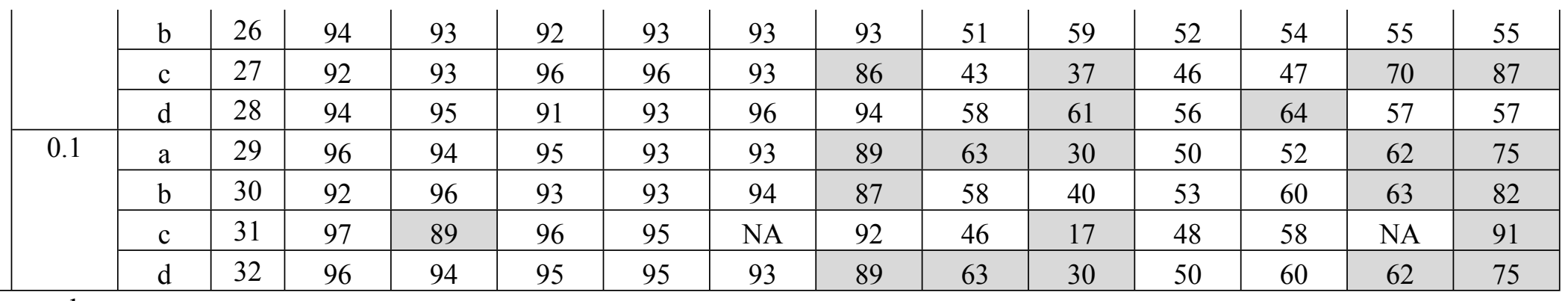
$a_{1}$ : Fixed-effect slope in the longitudinal submodel. $\lambda_{1}$ : Parameter for the effect of the time-dependent predictor on survival (true value $=-0.03$ ). NA: Not available due to convergence problems.

Refer to Figure 1 for other abbreviations.

691 Shading: $95 \%$ Confidence interval coverage $<90 \%$ or $\%$ estimates $<$ true value is outside the interval $[40,60]$. 
Table 3. Results of the estimation of $\lambda_{1}$ and $\tau$ from four sets of simulations for which group effect is non-zero.

\begin{tabular}{|c|c|c|c|c|c|c|c|c|c|c|c|c|c|}
\hline \multirow[b]{2}{*}{ Estimation of } & \multirow[b]{2}{*}{ Set } & \multicolumn{6}{|c|}{ Coverage of $95 \%$ confidence intervals } & \multicolumn{6}{|c|}{ Percentage of estimates $<$ true value } \\
\hline & & Cox.b & Cox.tp & joineR & stjm & JM.pc & JM.sp & Cox.b & Cox.tp & joineR & stjm & JM.pc & JM.sp \\
\hline \multirow{4}{*}{$\lambda_{1}$} & 33 & 84 & 96 & 97 & 97 & 92 & 94 & 11 & 22 & 55 & 61 & 95 & 80 \\
\hline & 34 & 92 & 93 & 97 & 94 & NA & 98 & 23 & 27 & 56 & 59 & NA & 79 \\
\hline & 35 & 83 & 92 & 94 & 96 & 83 & 68 & 20 & 26 & 54 & 58 & 90 & 95 \\
\hline & 36 & 89 & 76 & 91 & 93 & NA & 96 & 19 & 9 & 42 & 58 & NA & 84 \\
\hline \multirow{4}{*}{$\tau$} & 33 & 97 & 97 & 95 & 97 & 97 & 97 & 52 & 52 & 51 & 53 & 54 & 56 \\
\hline & 34 & 97 & 97 & 94 & 97 & NA & 97 & 52 & 49 & 51 & 50 & NA & 56 \\
\hline & 35 & 97 & 98 & 98 & 98 & 99 & 97 & 54 & 51 & 52 & 52 & 54 & 56 \\
\hline & 36 & 93 & 93 & 94 & 93 & NA & 95 & 49 & 50 & 49 & 50 & NA & 54 \\
\hline
\end{tabular}

$\lambda_{1}$ : Parameter for the effect of the time-dependent predictor on survival (true value $=-0.03$ ).

$695 \tau$ : Parameter for the group effect on survival (true value $=-0.5$ ).

696 Sets $33-36$ are the same as sets 3, 7, 19, 23 respectively in Table 2 except that the group effect is non-zero.

697 Refer to Figure 1 for the abbreviations.

698 Shading: 95\% Confidence interval coverage $<90 \%$ or $\%$ estimates $<$ true value is outside the interval [40, 60]. 
699 Table 4. Results of the estimation of $\lambda_{1}$ and $\tau$ from the real dataset.

\begin{tabular}{|l|c|c|c|c|c|c|}
\hline \multirow{2}{*}{ Method } & \multicolumn{3}{|c|}{ Estimation of $\lambda_{1}$} & \multicolumn{3}{c|}{ Estimation of $\tau$} \\
\cline { 2 - 7 } & estimate & se & p-value & estimate & se & p-value \\
\hline Cox.b & 0.065 & 0.0265 & 0.013 & 0.92 & 0.519 & 0.077 \\
\hline Cox.tp & 0.077 & 0.0226 & 0.001 & 0.77 & 0.523 & 0.139 \\
\hline joineR & 0.116 & 0.0425 & 0.006 & 1.00 & 0.600 & 0.095 \\
\hline stjm & 0.128 & 0.0463 & 0.006 & 0.58 & 0.512 & 0.257 \\
\hline JM.pc & 0.118 & 0.0448 & 0.008 & 0.56 & 0.511 & 0.276 \\
\hline JM.sp & 0.129 & 0.0478 & 0.007 & 0.69 & 0.544 & 0.203 \\
\hline JM.Cox & 0.114 & 0.0229 & $<0.00001$ & 0.67 & 0.501 & 0.184 \\
\hline
\end{tabular}

$700 \lambda_{1}$ : Parameter for the effect of the time-dependent predictor on survival (depression score).

$701 \tau$ : Parameter for the group effect on survival (family history of mental illness).

702 Refer to Figure 1 for method abbreviations.

703 